\title{
Les programmes de lutte contre le mariage des enfants : cerner le problème
}

Sajeda Amin

Population Council

Follow this and additional works at: https://knowledgecommons.popcouncil.org/departments_sbsr-pgy

Part of the Demography, Population, and Ecology Commons, Family, Life Course, and Society

Commons, Gender and Sexuality Commons, and the International Public Health Commons

How does access to this work benefit you? Let us know!

\section{Recommended Citation}

Amin, Sajeda. 2011. "Les programmes de lutte contre le mariage des enfants : cerner le problème,"

Promouvoir la santé, la sécurité et la productivité transitions vers l'âge adulte Bulletin no. 14. New York:

Population Council.

This Brief is brought to you for free and open access by the Population Council. 


\section{Les programmes de lutte contre le mariage des enfants: cerner le problème}

\section{Préparé par Sajeda Amin}

$$
\mathrm{L}
$$

e mariage des enfants est une violation des droits humains des filles et compromet leur santé et leur bien-être. En dépit de l'augmentation de l'âge du mariage dans la plupart des régions du monde en développement, le mariage précoce persiste au sein de nombreuses populations. L'on estime que dans le monde plus d'une femme sur trois âgées de 20 à 24 ans ont été mariées avant l'âge de 18 ans, et une femme sur sept a été mariée avant l'âge de 15 ans (analyse 2006 des données de l'Enquête démographique et de santé du Population Council). Les pratiques du mariage des enfants varient considérablement à travers et au sein des régions et entre groupes ethniques et religieux. Les plus fortes proportions de filles mariées sont généralement trouvées en Afrique occidentale et en Asie du Sud (voir le tableau 1 pour les régions les plus touchées par le mariage des enfants).

L'éradication du mariage des enfants est à l'ordre du jour des Nations unies et des pays individuels depuis fort longtemps. En effet, tous les pays où le mariage des enfants se produit sont signataires de chartes et de pactes internationaux qui en découragent la pratique. Les mécanismes internationaux relatifs aux droits humains, notamment la Déclaration universelle des droits de l'homme (1948), la Convention des Nations unies sur l'élimination de toutes les formes de discrimination à l'égard des femmes (1979), la Convention des Nations unies relative aux droits de l'enfant (1989), et la Charte africaine sur les droits et le bien-être de l'enfant (1990) proposent quatre protections fondamentales contre le mariage des enfants :

- L'exigence que les futures parties exercent un « consentement libre et total » dans la décision du mariage ;

- L'établissement de l'âge minimum du mariage à 18 ans, soit la limite supérieure de l'enfance telle que définie par la Convention relative aux droits de l'enfant ;

- L'appel aux États d'interdire « les pratiques néfastes », y compris le mariage des enfants ;

- Le droit des enfants à bénéficier de protections spéciales, y compris le droit d'exprimer librement leurs opinions dans toutes les questions
TABLEAU 1 Pourcentage de femmes âgées de 20 à 24 ans qui ont été mariées entre l'âge de 15 et 18 ans, par zone géographique

\begin{tabular}{lrrr} 
Zone & $\begin{array}{r}\text { Mariée avant } \\
15 \text { ans }\end{array}$ & $\begin{array}{r}\text { Mariée avant } \\
\text { 18 ans }\end{array}$ & $\begin{array}{r}\text { Âge moyen } \\
\text { au mariage }\end{array}$ \\
\hline Rajshahi, Bangladesh & 45 & 82 & 15 \\
Amhara, Ethiopie & 48 & 74 & 17 \\
Bihar, Inde & 18 & 60 & 18 \\
Guatemala rural & 13 & 53 & 19 \\
Sahel, Burkina Faso & 19 & 87 & 17 \\
Nord-ouest du Nigeria & 35 & 76 & 17 \\
\hline
\end{tabular}

Source : Population Council. 2011.

concernant leur bien-être ; le droit à l'éducation sur la base de l'égalité des chances ; le droit à la protection contre toutes les formes de violence physique ou mentale, blessures ou abus, maltraitance ou exploitation ; et le droit à la santé et l'accès aux services de santé.

Malgré ces accords, le mariage avant l'âge de 18 ans persiste et souvent sans le consentement de la jeune mariée. Un certain nombre de facteurs sous-jacents contribuent à perpétuer le mariage précoce : les normes de genre traditionnelles, la grande valeur accordée à la virginité féminine, les préoccupations des parents entourant les rapports sexuels et la grossesse avant le mariage, les pressions dues à la dot, la perception que le mariage offre une protection contre le $\mathrm{VIH}$ et les autres infections sexuellement transmissibles, et le souhait d'assurer des alliances sociales, économiques ou politiques. La pauvreté joue également un rôle majeur. Une famille très pauvre est susceptible de penser que la famille du mari sera mieux placée pour subvenir aux besoins de leur fille ou que les gains économiques engendrés par son mariage peuvent faire partie de la stratégie de survie de la famille.

Des travaux de recherche suggèrent que ces facteurs se rejoignent autour des opportunités économiques et éducatives des filles. Par exemple, l'association entre le mariage des enfants et les faibles niveaux de scolarisation est toujours forte dans toutes les régions du 
monde en développement. Les femmes ayant trois années ou moins de scolarité sont beaucoup plus susceptibles de s'être mariées plus tôt que celles qui ont huit années de scolarité ou plus (Mensch 2005). Le fait d'avoir quitté l'école est moins susceptible d'être une conséquence du mariage précoce. Le plus souvent, les mêmes conditions sous-jacentes qui dictent la décision du moment du mariage - la pauvreté, un statut faible et des normes culturelles - sont responsables du désinvestissement dans les filles et limitent leur éducation (Mensch 2005). Par ailleurs, l'éducation des femmes et le moment du mariage sont des éléments associés à travers les générations. Selon une étude menée au Bangladesh, par exemple, des niveaux élevés d'éducation maternelle sont associés au fait de marier les filles plus tard (Bates et al. 2007). Outre l'école, on observe une association cohérente entre le mariage précoce et la participation des femmes à la vie active avant le mariage. Les résultats d'études menées au Bangladesh montrent que les jeunes travailleuses du vêtement se marient beaucoup plus tard que les filles qui ne font pas partie de la population active (Amin et al. 1998).

Ces données suggèrent que les possibilités éducatives et économiques offrent aux jeunes femmes des alternatives positives au mariage précoce qui sont acceptables pour la famille et à la communauté.

Les actions visant à mettre fin au mariage des enfants L'omniprésence du mariage des enfants nécessite la conduite d'actions multisectorielles pour recentrer la transition vers le mariage. Un tel recadrage comprend trois objectifs complémentaires :

- Retarder l'âge du mariage ;

- Rendre le processus du mariage consensuel, plus sûr et plus équitable ; et

- Fournir un soutien aux filles mariées (Haberland 2007).

Les chercheurs du Population Council ont pour objectif d'empêcher le mariage des enfants et de soutenir les filles mariées dans plusieurs pays, dont le Bangladesh, le Burkina Faso, l'Égypte, l'Éthiopie, le Guatemala, I'Inde, le Kenya, le Nigéria et le Vietnam. Ces programmes comprennent des opportunités en expansion (l'éducation, les moyens de subsistance) pour les filles, en particulier celles qui sont le plus à risque d'un mariage précoce, et un travail direct avec les tuteurs (parents, garçons / hommes, chefs religieux et communautaires) pour éliminer les obstacles qui empêchent les filles d'accéder à ces opportunités. De telles actions peuvent être, par exemple, des messages médiatiques de masse et des rencontres entre filles et tuteurs pour étudier les causes et les conséquences du mariage précoce et / ou fournir des incitations économiques pour retarder l'âge du mariage.

Les efforts déployés pour rendre le processus du mariage plus sûr comprennent des conseils et un dépistage volontaire pour les jeunes femmes et hommes qui envisagent de se marier, en favorisant le consentement plus significatif des filles au cours du processus du mariage, et en encourageant des décisions mieux informées par la famille de la mariée. Dans certains contextes, le fait d'agir sur les facteurs économiques, tels que la dot et les coûts du mariage, est une solution. Ces facteurs économiques sont une préoccupation, non seulement parce qu'ils représentent les causes du mariage précoce, mais également parce que ces transactions mettent les filles en danger. Par exemple, les jeunes femmes au Bangladesh qui ont payé une dot sont significativement plus susceptibles de déclarer avoir été abusées pendant l'année écoulée, et celles qui ont payé une dot après le mariage ont de plus grandes chances d'avoir été battues que celles qui n'en avaient pas payé (Suran et al. 2004).

Kishori Abhijan : Autonomiser les filles en leur donnant l'accès aux moyens de subsistance pour retarder le mariage au Bangladesh Au Bangladesh, 68 pour cent des filles de plus de 18 ans ont été mariées avant leur $18^{\mathrm{e}}$ anniversaire, et une fille sur trois a été mariée avant l'âge de 15 ans. Bien qu'il y ait de grandes variations dans le pays, des enquêtes récentes suggèrent que près de la moitié des mariages entraînent le paiement de dots substantielles par la famille de la fille à la famille du marié. La majorité des mariages sont arrangés, et, bien que la plupart des filles déclarent avoir donné leur consentement, conformément à la loi islamique, quatre filles sur cinq rencontrent leur mari le jour du mariage. Ces statistiques sont particulièrement déroutantes, si l'on tient compte des énormes progrès qui ont été réalisés en matière de scolarisation primaire et secondaire des filles, de la baisse spectaculaire de la fécondité chez les femmes mariées et de l'élargissement des programmes de micro crédit qui répondent en priorité aux femmes des zones rurales. La poursuite du mariage précoce va à l'encontre des nombreux aspects positifs observés dans d'autres secteurs.

Dans le but d'inverser ces tendances, le Population Council s'est associé avec deux organisations non gouvernementales (ONG) bangladaises pour concevoir et évaluer un programme à large échelle consacré aux adolescentes. Ce programme, appelé Kishori Abhijan, est axé sur la création d'espaces sûrs permettant de pousser les filles à chercher des alternatives au mariage précoce. II proposait un espace sûr permettant aux filles de se rencontrer, des leçons sur les compétences de vie et dans certains cas, l'accès à une formation destinée à améliorer les compétences de subsistance, les possibilités d'épargne et de crédit. Au bout de deux ans, le programme a réussi à retarder le mariage chez les filles les plus pauvres et les plus jeunes. Mais sachant que les filles doivent encore payer une dot et que les filles plus âgées doivent payer une dot plus élevée, le montant de la dot moyenne versée parmi celles qui ont retardé le mariage a augmenté. Les responsables du programme ont conclu que les efforts visant à retarder le mariage sont les plus bénéfiques quand ils élargissent les débouchés économiques en faveur des filles, qui considèrent ces nouvelles possibilités comme des alternatives significatives au mariage précoce. Ces possibilités contribuent également à défrayer les coûts croissants de la dot que le mariage tardif entraîne (Amin et Suran, 2005a, b).

Berhane Hewan : Retarder le mariage précoce chez les filles défavorisées en milieu rural dans la région Amhara, en Éthiopie, grâce au soutien social, à l'éducation et à la sensibilisation des communautés Dans la région Amhara en Éthiopie, les taux de mariages d'enfants sont parmi les plus élevés au monde. La moitié de toutes les filles dans la ré-

2 - Rendez-vous sur www.popcouncil.org/publications/serialsbriefs/TABriefs.asp pour consulter tous les bulletins d'information de la série Promoting healthy, safe, and productive transitions to adulthood [Promouvoir des transitions saines, sécurisées et productives vers l'âge adulte]. 
gion Amhara sont mariées avant leur $15^{\mathrm{e}}$ anniversaire. Une étude menée par le Population Council dans la région Amhara et à Addis-Abeba a révélé que pour beaucoup de jeunes filles le mariage peut signifier avoir des relations sexuelles avec un inconnu : 95 pour cent des filles interrogées ne connaissaient pas leur mari avant le mariage, et 85 pour cent n'ont pas été informées qu'elles étaient sur le point de se marier. Plus des deux tiers des filles mariées ont déclaré qu'elles n'avaient même pas encore eu leurs règles quand elles ont eu leur premier rapport sexuel (Erulkar et al. 2004).

Berhane Hewan (qui signifie « Lumière pour Ėve » en amharique) est un programme conçu pour aider les filles en leur apportant les connaissances, les compétences et les ressources nécessaires pour éviter le mariage d'enfant. Le programme encourage l'alphabétisation fonctionnelle, les compétences de vie et l'éducation sur la santé reproductive à travers des groupes de filles mobilisées et dirigées par une tutrice. Reconnaissant les raisons économiques qui encouragent le mariage des enfants, des incitations matérielles ont été ajoutées pour encourager les familles à laisser leurs filles participer aux groupes et rester à l'école. Le programme a été évalué après 18 mois. Les résultats suggèrent que, comparativement aux niveaux de mariage observés au début du programme, nettement moins de filles âgées de 10 à 14 ans se sont mariées (1,6 pour cent dans la zone d'étude contre 22,1 pour cent dans la zone témoin), et un nombre significatif de filles plus jeunes sont scolarisées (Erulkar et Muthengi 2009).

\section{Abriendo Oportunidades : Développement des ressources} économiques, sociales et sanitaires pour éviter le mariage précoce au Guatemala

Le Guatemala est l'un des pays les plus diversifiés en Amérique latine au niveau ethnique. C'est également l'une des zones les plus pauvres de l'hémisphère occidental, sachant que la pauvreté concerne principalement les groupes mayas. Les filles mayas sont le groupe de population le plus défavorisé du pays ; l'abandon scolaire précoce, le mariage précoce, les grossesses précoces et non désirées, l'analphabétisme, et les possibilités de vie limitées sont toutes des caractéristiques communes de leur transition vers l'âge adulte. Près de 40 pour cent des filles mayas sont mariées avant l'âge de 18 ans.

En 2004, le Population Council a lancé un projet intitulé Abriendo Oportunidades conçu pour aider les filles à faire une transition réussie vers l'âge adulte, y compris retarder le mariage. Des filles mayas âgées de 16 à 20 ans ont été sélectionnées pour participer à des ateliers sur des thèmes tels que le leadership, les compétences professionnelles, la prise de parole en public, les relations entre les sexes et la santé sexuelle et reproductive. Des filles « leaders » provenant de groupes d'ONG locales ont formé des filles plus jeunes (10-15 ans) dans leurs communautés pour qu'elles deviennent tutrices et ont été formées sur l'estime de soi, les compétences de vie et la santé reproductive. Le projet a ainsi créé des « espaces sûrs » pour les filles mayas et a développé le sens de la communauté parmi elles. En plus d'acquérir de précieuses connaissances pratiques, de nombreuses jeunes filles ont également pu articuler leurs projets d'avenir, tels que la poursuite de leurs études et l'ouverture de leurs propres entreprises.
Le mariage précoce chez les Hmong et les Dao dans les hauts plateaux du Nord Vietnam

Au Vietnam, la croissance rapide de l'économie a bénéficié à certains groupes, tels que la population majoritaire Kinh, tout en laissant les autres derrière. Avec faible niveau d'éducation et de santé, le mariage précoce est l'une des caractéristiques déterminantes de deux des groupes minoritaires les plus défavorisés, les Hmong et les Dao.

Les chercheurs du Population Council ont mené une étude dans le quartier pauvre et éloigné de Vo Nhai dans la province de Thai Nguyen, où les Dao et les Hmong vivent avec leurs voisins, les Kinh. Les chercheurs ont appris que les Kinh sont susceptibles de trouver des opportunités d'éducation et d'emploi en dehors de leur commune et ont tendance à retarder le mariage. En revanche, plus de 65 pour cent des hommes et des femmes hmong et dao âgées de 20 à 25 ans sont mariés. Le mariage est un moyen important d'obtenir des familles natales l'indépendance économique et une reconnaissance en tant qu'adulte (Amin et Teerawichitchainan 2009).

\section{Pistes de travail}

Les variations importantes dans les pratiques du mariage, au sein même des pays et des communautés, suggèrent que notre compréhension du processus du mariage reste limitée. On sait peu de choses, par exemple, sur le consentement au mariage, le choix du conjoint, la familiarité avec le conjoint choisi et les transactions matrimoniales. II existe des preuves montrant que les jeunes femmes prennent de plus en plus en charge le processus du mariage, ce qui pourrait être associé à la baisse des mariages précoces. Nous émettons l'hypothèse que ces caractéristiques du mariage interagissent avec la pauvreté et l'autonomie des femmes dans la famille du marié et dans la communauté pour influencer le bien-être et la santé sexuelle et reproductive des femmes et des filles.

Les programmes et les travaux de recherche futurs doivent chercher à clarifier et à répondre aux pressions exercées sur les familles pour marier leurs filles précocement; examiner si et comment le mariage - ou les composantes spécifiques du processus du mariage (comme le consentement, la dot, l'âge au mariage) - ne contribue pas à faire perdre aux femmes leurs relations intimes en les exposant au risque d'effets négatifs sur la reproduction et la santé ; tester la façon dont ces effets peuvent être atténués ; et améliorer la transition vers le mariage. Par exemple, le droit de pouvoir consentir au mariage peut-il être plus pertinent dans le contexte des filles, s'agissant de choisir le marié et le moment du mariage ? Et comment un consentement valable peut-il offrir des alternatives dans des environnements où le mariage précoce est établi et où il n'existe en général peu ou pas d'alternatives?

Les travaux de recherche du Population Council montrent que les programmes qui fonctionnent pour les filles sont basés sur une compréhension claire du contexte culturel et économique local. Ces programmes visent également à élargir les possibilités pour les filles et limiter les contraintes à l'autonomisation économique qui obligent les filles et leurs familles à choisir le mariage et les grossesses précoces. 


\section{Bibliographie et publications connexes}

Amin, Sajeda. 2011. "Empowering adolescent girls in rural Bangladesh: Kishori Abhijan," Promoting Healthy, Safe, and Productive Transitions to Adulthood Brief no. 13. New York: Population Council.

Amin, Sajeda et Ashish Bajracharya. 2011. "Costs of Marriage - Marriage Transactions in the Developing World," Promoting Healthy, Safe, and Productive Transitions to Adulthood Brief no. 37. New York: Population Council.

Amin, Sajeda and Bussarawan Teerawichitchainan. 2009. "Ethnic Fertility Differentials in Vietnam and Their Proximate Determinants." Document de travail $n^{\circ} 18$ de la Division de la recherche en politiques du Population Council. New York: Population Council.

Amin, Sajeda. 2006. "The implications of trade liberalization for working women's marriage: Case studies of Bangladesh, Egypt and Vietnam," dans Caren Grown, Elissa Braunstein, et Anju Malhotra (éds.), Trading Women's Health and Rights? Trade Liberalization and Reproductive Health in Developing Economies. London: Zed Books, pp. 97-120.

Amin, Sajeda, Nasheeba Selim, et Nashid K. Waiz. 2006. "Causes and consequences of early marriage in Bangladesh: Background report for workshop on programs and policies to prevent early marriage." Dhaka: Population Council.

Amin, Sajeda. 2004. "Children and adolescents in Bangladesh: Definitions, rights and realities," in Dina M. Siddiqi (ed.), Human Rights in Bangladesh: 2003. Dhaka: Ain-o-Shalish Kendra.

Amin, Sajeda et Luciana Suran. 2005a. "Program efforts to delay marriage through improved opportunities: Some evidence from rural Bangladesh." Document présenté lors de la Réunion annuelle de la Population Association of America, Philadelphia, 31 mars-2 avril.

Amin, Sajeda et Luciana Suran. 2005b. "The impact of marriage payments on leisure, housework and abuse of young wives: Some evidence from rural Bangladesh." Document présenté lors de la Réunion annuelle de la Population Association of America, Philadelphia, 31 mars-2 avril.

Amin, Sajeda, lan Diamond, Ruchira Naved, et Margaret Newby. 1998. "Transition to adulthood of female garment-factory workers in Bangladesh," Studies in Family Planning 29(2): 185-200.

Bates, Lisa, Joanna Maselko, et Sidney Ruth Schuler. 2007. "Women's education and the timing of marriage and childbearing in the next generation: Evidence from rural Bangladesh," Studies in Family Planning 38(2): 101-112.

Catino, Jennifer, Alejandra Colom et Marta Julia Ruiz. 2011. "Equipping Mayan girls to improve their lives," Promoting Healthy, Safe, and Productive Transitions to Adulthood Brief no. 5. New York: Population Council.

Clark, Shelley, Judith Bruce, et Annie Dude. 2006. "Protecting young women from HIVIAIDS: The case against child and adolescent marriage," International Family Planning Perspectives 32(2): 79-88.

Erulkar A.S. et Muthengi E. 2009. "Evaluation of Berhane Hewan: A program to delay child marriage in rural Ethiopia". Int Perspect Sex Reprod Health, 35(1):6-14

Erulkar, Annabel et Francis Ayuka. 2007. "Addressing early marriage in areas of high HIV prevalence: A program to delay marriage and support married girls in rural Nyanza, Kenya." New York: Population Council.

Erulkar, Annabel, et Tekle Ab Mekbib. 2007. "Reaching disadvantaged rural girls, creating social support, and discouraging child marriage in Amhara, Ethiopia." New York: Population Council.
Erulkar, Annabel, Tekle Ab Mekbib, Negussie Simie, et Tsehai Gulema. 2004. "The experience of adolescence in rural Amhara region, Ethiopia." Accra, Ghana: Population Council.

Glynn, J.R., M. Caraël, B. Auvert, M. Kahindo, J. Chege, R. Musonda, F. Kaona, A. Buvé, and the Study Group on Heterogeneity of HIV Epidemics in African Cities. 2001. "Why do young women have a much higher prevalence of HIV than young men? A study in Kisumu, Kenya and Ndola, Zambia," AIDS 15(supplement 4): S51-S60.

Hallman, Kelly, Eva Roca, Marta Julia Ruiz, Jennifer Catino, Alejandra Colom,et Sandra Contreras Aprile. 2007. "For Mayan girls, safe spaces lead to social gains." New York: Population Council.

Haberland, Nicole 2007. "Supporting married girls: Calling attention to a neglected group," Transitions to Adulthood Brief No. 3. New York: Population Council.

Mensch, Barbara S. 2005. "The transition to marriage," in Growing Up Global: The Changing Transitions to Adulthood in Developing Countries. Panel on Transitions to Adulthood in Developing Countries, Cynthia B. Lloyd (ed.). Washington, DC: The National Academies Press, pp. 416-505.

Mensch, Barbara, Monica Grant, et Ann Blanc. 2005. "The changing context of sexual initiation in sub-Saharan Africa," document présenté lors de la reunion de l'Union internationale pour l'étude scientifique de la population, Tours, 18-23 juillet.

Mensch, Barbara, Susheela Singh, et John Casterline. 2005. "Trends in the timing of first marriage among men and women in the developing world," in The Changing Transitions to et in Developing Countries: Selected Studies, Cynthia B. Lloyd, Jere R. Behrman, Nelly P. Stromquist, and Barney Cohen (eds.). Washington, DC: The National Academies Press.

Population Council. 2011. "The Adolescent Experience In-depth: Using Data to Identify and Reach the Most Vulnerable Young People," http://www.popcouncil. org/publications/serialsbriefs/AdolExplnDepth.asp. Consulté le 27 mai 2011.

Santhya, K.G. et Shireen Jejeebhoy. 2003. "Sexual and reproductive health needs of married adolescent girls," Economic and Political Weekly 38(41): 4370-4377.

Santhya, K.G., Nicole Haberland, et Ajay Kumar Singh. 2006. "'She only knew when the garland was put around her neck': Findings from an exploratory study on early marriage in Rajasthan." New Delhi: Population Council.

Santhya, K.G. et Shireen J. Jejeebhoy. 2007. "Early marriage and HIVIAIDS: Risk factors among young women in India," Economic and Political Weekly 42(14): 1291-1297.

Suran, Luciana, Sajeda Amin, Lopita Huq, et Kobita Chowdury. 2004. "Does dowry improve life for brides? A test of the bequest theory of dowry in rural Bangladesh." Document de travail $n^{\circ} 195$ de la Division de la recherche en politiques du Population Council. New York: Population Council.

\section{Bailleurs de fonds}

Au cours de ces dernières années, les bailleurs de fonds ont été : le Département pour le développement international (UK), la Fondation Ford, la Fondation Bill \& Melinda Gates, la Fondation Libra, la Fondation John D. et Catherine T. MacArthur, la Fondation Rapidan, la Fondation Turner, le Fonds des Nations unies pour l'enfance (UNICEF), le Fonds des Nations unies pour la population (UNFPA), l'Agence américaine pour le développement international (USAID) et l'Organisation mondiale de la santé.

\section{Population Council}

Le Population Council fait évoluer les perspectives sur les questions essentielles de santé et de développement. Nous cherchons à comprendre les causes et les conséquences de l'inégalité entre les sexes et des disparités sociales qui s'installent au cours de l'adolescence. Nous fournissons des données en vue d'améliorer les programmes et les politiques mis en place sur le terrain qui permettent d'assurer des transitions efficaces et productives vers la vie adulte dans les pays en développement. www.popcouncil.org

(C) 2011 The Population Council, Inc.

4 - Rendez-vous sur www.popcouncil.org/publications/serialsbriefs/TABriefs.asp pour consulter tous les bulletins d'information de la série Promoting healthy, safe, and productive transitions to adulthood [Promouvoir des transitions saines, sécurisées et productives vers l'âge adulte]. 